\title{
Direct Numerical Simulation of Transition in a Swept-Wing Boundary Layer
}

\author{
Lian Duan* \\ National Institute of Aerospace, Hampton, VA 23666 \\ Meelan M. Choudhari ${ }^{\dagger}$, Fei $\mathrm{Li}^{\ddagger}$ \\ NASA Langley Research Center, Hampton, VA 23681
}

\begin{abstract}
Direct numerical simulation (DNS) is performed to examine laminar to turbulent transition due to high-frequency secondary instability of stationary crossflow vortices in a subsonic swept-wing boundary layer for a realistic natural-laminar-flow airfoil configuration. The secondary instability is introduced via inflow forcing derived from a two-dimensional, partial-differential-equation based eigenvalue computation; and the mode selected for forcing corresponds to the most amplified secondary instability mode which, in this case, derives a majority of its growth from energy production mechanisms associated with the wall-normal shear of the stationary basic state. Both the growth of the secondary instability wave and the resulting onset of laminar-turbulent transition are captured within the DNS computations. The growth of the secondary instability wave in the DNS solution compares well with linear secondary instability theory when the amplitude is small; the linear growth is followed by a region of reduced growth resulting from nonlinear effects before an explosive onset of laminar breakdown to turbulence. The peak fluctuations are concentrated near the boundary layer edge during the initial stage of transition, but rapidly propagates towards the surface during the process of laminar breakdown. Both time-averaged statistics and flow visualization based on the DNS reveal a sawtooth transition pattern that is analogous to previously documented surface flow visualizations of transition due to stationary crossflow instability. The memory of the stationary crossflow vortex is found to persist through the transition zone and well beyond the location of the maximum skin friction.
\end{abstract}

\section{Nomenclature}

$A \quad$ instability amplitude measured in terms of peak fluctuation in chordwise velocity, $\mathrm{m} / \mathrm{s}$

$c \quad$ wing chord length measured in direction perpendicular to leading edge, $\mathrm{m}$

$c_{s} \quad$ oblique wing-chord length measured in direction parallel to the freestream direction, $\mathrm{m}$

$C_{f} \quad$ skin friction coefficient, dimensionless

$f \quad$ frequency of instability oscillations, $\mathrm{Hz}$

$T K E$ turbulent kinetic energy, $\mathrm{m}^{2} / \mathrm{s}^{2}$

$M \quad$ Mach number, dimensionless

$R e_{c_{s}}$ Reynolds number based on the oblique wing-chord length along the freestream direction, $R e_{c s} \equiv \rho_{\infty} U_{\infty} c_{s} / \mu_{\infty}$, dimensionless

$U_{\infty}$ freestream velocity, $\mathrm{m} / \mathrm{s}$

$T$ temperature, $\mathrm{K}$

$T_{r} \quad$ recovery temperature, $T_{r}=T_{\infty}\left(1+0.9 \frac{\gamma-1}{2} M_{\infty}^{2}\right), \mathrm{K}$

$u_{\tau}$ friction velocity, $\mathrm{m} / \mathrm{s}$

\footnotetext{
${ }^{*}$ Research Scientist. Member, AIAA

$\dagger$ Aerospace Technologist, Computational AeroSciences Branch, M.S. 128. Associate Fellow, AIAA

¥Aerospace Technologist, Computational AeroSciences Branch, M.S. 128
} 
$u_{x} \quad$ chordwise velocity (perpendicular to leading edge), $\mathrm{m} / \mathrm{s}$

$u_{y} \quad$ spanwise velocity (parallel to leading edge), $\mathrm{m} / \mathrm{s}$

$u_{z} \quad$ wall-normal velocity, $\mathrm{m} / \mathrm{s}$

$x \quad$ wing surface coordinate in the direction perpendicular to the leading edge of the swept wing

$y \quad$ wing surface coordinate in the direction parallel to the leading edge of the swept wing

$z \quad$ coordinate normal to the wing surface

$z_{\tau} \quad$ viscous length, $z_{\tau}=\nu_{w} / u_{\tau}, \mathrm{m}$

$i \quad$ wing surface coordinate along the axis of stationary crossflow vortex

$j \quad$ wing surface coordinate parallel to the leading edge in the non-orthogonal system

$\lambda_{y} \quad$ spanwise wavelength of crossflow instability in direction parallel to leading edge, $\mathrm{mm}$

$\Lambda \quad$ wing sweep angle, degree

\begin{tabular}{ll}
\multicolumn{2}{l}{ Subscripts } \\
$i$ & inflow station for the domain of direct numerical simulations \\
max & maximum value \\
rms & root mean square \\
$\mathrm{w}$ & wall variables \\
$\infty$ & freestream variables \\
Superscripts \\
$\overline{+} \quad$ inner wall units \\
$(\cdot) \quad$ averaged variables \\
$(\cdot)^{\prime} \quad$ perturbation from averaged variable
\end{tabular}

\section{Introduction}

Skin-friction drag accounts for approximately one-half of the total drag for a transport aircraft. ${ }^{1}$ Therefore, reduction in skin-friction drag can lead to substantial improvement in fuel efficiency for future aircraft. A laminar boundary layer has significantly lower skin friction than a turbulent boundary layer at the same condition. Therefore, flow control via delayed boundary-layer transition over major aerodynamic surfaces holds the potential to provide the desired reductions in the overall drag. One promising strategy for Laminar Flow Control (LFC) over a swept aerodynamic surface is to use control input in the form of appropriately positioned Discrete Roughness Elements (DREs) near the leading edge. ${ }^{2}$ The bulk of existing experimental and computational studies of the DRE concept have been carried out for either incompressible or supersonic configurations with modest wing-chord Reynolds numbers of up to approximately 8 million, ${ }^{3,4}$ and with pressure distributions that may not be optimal for wing designs for subsonic transport aircraft flying at Mach numbers between 0.75 to 0.90 . Consequently, additional effort is necessary to assess the potential capability of the DRE concept to control swept-wing transition at transonic Mach numbers and substantially higher chord Reynolds numbers than previous applications.

Recently, Texas A\&M University ${ }^{5}$ (TAMU) performed a conceptual design study of an LFC flight test with target conditions of $M_{\infty}=0.75$ at an altitude of $40,000 \mathrm{ft}$, a chord Reynolds number, $R e_{c}$, between 15 and 20 million, and a leading-edge sweep angle, $\Lambda$, of $30^{\circ}$. The resulting wing had a significant potential for natural laminar flow over both suction and pressure surfaces at the design angle-of-attack equal to zero degrees. At off-design conditions, however, an earlier transition may occur as a result of stationary crossflow modes, which opens up the possibility of roughness-based transition control on the same model, either to increase the lift coefficient or to extend the angle-of-attack range for laminar flow. ${ }^{5}$ The designed wing configuration was also studied numerically at NASA Langley Research Center ${ }^{6}$ to assess the feasibility of achieving DRE-based transition control with NASA developed prediction tools pertaining to different stages of the transition process. In particular, $\mathrm{Li}$ et al. ${ }^{6}$ investigated the linear amplification of most unstable stationary crossflow modes, the nonlinear development of individual stationary modes, and the linear growth of high-frequency secondary instablities for the laminar flow airfoil at aforementioned off-design conditions. They also investigated the initial stage during the breakdown of crossflow vortices with nonlinear parabolized stability equations (PSE).

Direct numerical simulation (DNS) is an important ingredient in the hierarchical prediction approach, which can be used to study complete transition due to crossflow vortices and understand the breakdown 
mechanisms responsible for the onset of transition. DNS is crucial to guiding and validating the application of lower fidelity tools in complex 3D flows and will eventually serve as a proxy to controlled experiments in a fully quantifiable disturbance environment. Earlier DNS of boundary layers have concentrated either on the linear and early nonlinear regime or the fully developed turbulent flows. The few more recent DNS studies that simulate the entire transition regime to a fully turbulent boundary layer include those of bypass transition in a nominally-zero-pressure-gradient incompressible boundary layer over a smooth flat plate $^{7}$ and transition via oblique breakdown in a two-dimensional supersonic boundary layer. ${ }^{8,9}$ In terms of complete transition in a three-dimensional boundary layer due to stationary crossflow vortices, Wassermann and Kloker ${ }^{10}$ carried out DNS of secondary instability well into the breakdown region and revealed some interesting features of the breakdown process for an incompressible, flat-plate boundary layer. However, since disturbance statistics in the fully turbulent region was not compared with experiments and well-established simulations of fully turbulent wall bounded flows, it is not clear whether or not turbulence had fully developed in their DNS. Moreover, they introduced unsteady disturbances via a disturbance strip at the wall, which eventually resulted in a 'Z-mode' secondary instability. As a result, the computed breakdown features were limited to those associated with the Z-mode instabilities. However, linear and nonlinear PSE analyses ${ }^{6,11}$ indicate that, if the Y-modes are excited in certain flows, then they would achieve a considerable spatial growth and, indeed, can lead to transition under appropriate circumstances. Here, the letter notation for different classes of secondary instability modes follows the suggestion of Malik et al. ${ }^{12}$ with 'Z-mode' denoting the high-frequency 'mode I' induced by the spanwise gradient of the streamwise velocity component and ' $\mathrm{Y}$ mode' denoting the high-frequency 'mode II' induced by the local maximum of the wall-normal gradient. The current DNS is complementary to previously reported simulations by Wassermann and Kloker ${ }^{10}$ and focuses on the Y-mode of secondary instability in the swept wing boundary layer corresponding to the TAMU configuration $^{5}$ and its subsequent breakdown to turbulence. Such a simulation is necessary to answer the question of whether or not the Y-mode of secondary instability leads to a fully turbulent boundary layer. In addition, the turbulence statistics in the fully turbulent regime can be compared with experiments and well-established turbulent simulations to ensure that a fully developed turbulent boundary layer is reached.

This paper is structured as follows. The flow conditions and numerical methods are outlined in Section II. Section III presents DNS results of secondary instability and laminar breakdown. Concluding remarks are presented in Section IV.

\section{Flow Conditions and Numerical Methodology}

Freestream conditions for the work described in this paper are summarized in Table 1. The mean boundary-layer flow over the suction surface of the airfoil is computed with a boundary layer solver using the infinite span approximation, in conjunction with the inviscid surface pressure distribution derived from an Euler solution under the free flight conditions. Linear and nonlinear development of the instability modes is computed using parabolized stability equations as implemented in the Langley Stability and Transition Analysis Codes (LASTRAC). ${ }^{13}$ The current DNS focuses on the nonlinear evolution and breakdown of the stationary crossflow vortex with spanwise wave length $\lambda_{y}=8 \mathrm{~mm}$. The details of the methodology employed for the mean boundary-layer flow over the suction surface of the airfoil, the linear and nonlinear development of the instability modes, and the secondary instability predictions are summarized in Li et al. ${ }^{6}$

Table 1. Freestream conditions for the DNS.

\begin{tabular}{cccccc}
\hline \hline$M_{\infty}$ & $U_{\infty}(\mathrm{m} / \mathrm{s})$ & $\rho_{\infty}\left(\mathrm{kg} / \mathrm{m}^{3}\right)$ & $T_{\infty}(\mathrm{K})$ & $c(\mathrm{~m})$ & $R e_{c s}\left(\times 10^{6}\right)$ \\
\hline 0.75 & 221.28 & 0.302 & 216.65 & 3.1676 & 17.2 \\
\hline \hline
\end{tabular}

A non-orthogonal, vortex aligned grid is used for the current DNS. In this coordinate system, the streamwise computational coordinate $i$ is oriented along the direction of the crossflow vortex axis (orthogonal to 
the wave number vector $(\alpha, \beta)$ in the $x-y$ plane) rather than along the chordwise direction ( $x$ direction). By doing so, fewer grid points are required in the streamwise direction to resolve the evolution of stationary crossflow modes. The spanwise computational coordinate $j$ is along the $y$ direction which is parallel to the leading edge of the wing and is inclined at approximately sixty degrees with respect to the $i$-coordinate. Fig. 1 shows a schematic consisting of a periodic pattern of stationary crossflow vortices as seen from above the airfoil surface, along with a black frame depicting the boundaries of the DNS domain along the $i$ and $j$ directions. Similar non-orthogonal systems have been chosen in spatially developing secondary instability analyses ${ }^{14}$ and $\mathrm{DNS}^{15}$ for crossflow-dominated swept-wing boundary layers.

The computational domain extends from $50.0 \%<x / c<57.2 \%$ in the streamwise direction, which covers the entire transition region starting from the nearly stationary flow upstream to a small region of nascent turbulence near the downstream end. In the spanwise direction the domain is limited to a single wavelength, $\lambda_{y}$, of the primary stationary crossflow vortex. Uniform grid spacings are used in the streamwise $(i)$ and spanwise $(j)$ computational coordinates, with a grid density of 40 and 180 points per wavelength, respectively. This grid resolution corresponds to approximately $\Delta x^{+}=8.6$ and $\Delta y^{+}=5.2$ in the fully turbulent region, based on the viscous length scale $z_{\tau}$ at a reference post-transition location $x_{r e f}=1.8 \mathrm{~m}$ ( or $x_{r e f} / c=56.9 \%$ ), which is close to the exit of the DNS domain. The wall-normal domain size is about $18.5 \delta_{\text {ref }}$ with clustering of grid points both near the wall and in the outer part of the boundary layer. This wall-normal grid clustering is designed to capture both secondary instability near the edge of the boundary layer and turbulence dynamics near the wall. The total number of grid points is 3,200, 180, and 300 in the streamwise $(i)$, spanwise $(j)$, and wall-normal $(k)$ directions, respectively. The computational grid resolution in the post-transition regime is comparable to those reported in the literature for simulations of fully turbulent wall-bounded flows with comparable numerical algorithms. ${ }^{16-19}$

To simulate the boundary layer flow over the domain, the compressible Navier-Stokes equations are solved in generalized curvilinear coordinates. The working fluid is assumed to be an ideal gas with a linear (i.e. Newtonian) stress-strain relation. Fouriers law is used to compute the heat flux terms, and a 7th-order weighted essentially non-oscillatory (WENO) scheme $\mathrm{e}^{20,21}$ is used to compute the convective flux terms. This particular WENO algorithm combines a high order of accuracy with low dissipation and high bandwidth, which is desirable for time-accurate simulations of compressible turbulence. For the viscous flux terms, a 4th-order central difference scheme is used. The 3rd-order low-storage Runge-Kutta scheme by Williamson ${ }^{22}$ is used for time integration.

At the inlet, a three-dimensional laminar profile corresponding to the finite-amplitude stationary crossflow vortex is specified as the basic state (Fig. 2a), which is superimposed by the selected Y-mode secondary instability wave as disturbances (Fig. 2b). For the outflow boundary, a buffer domain technique ${ }^{23,24}$ is used to avoid reflections of disturbance waves from the outflow boundary layer. The length of the buffer domain is approximately four times the streamwise wavelength of fundamental disturbances in the linear regime. On the wall, no-slip conditions are applied for the three velocity components and an isothermal condition is used for the temperature, with the wall temperature prescribed to be equal to the laminar adiabatic temperature $T_{r}$. At the upper boundary, characteristic non-reflecting boundary conditions based on Thompson ${ }^{25}$ are imposed. Periodic boundary conditions are used in the spanwise direction.

\section{Results}

Figure 3 shows the streamwise evolution of the fundamental modal amplitude of the secondary instability mode. The modal amplitude is measured in terms of the peak streamwise velocity perturbation over the local cross-section of the underlying crossflow vortex. The DNS results based on two separate parts of the simulation interval (depicted by red and green curves) are compared with the predictions of linear secondary instability theory and a linear PSE calculation reported by Li et al. ${ }^{6}$ The DNS result indicated by the red curve corresponds to a Fourier transform over a time history of more than one hundred periods of the fundamental mode, whereas the green curves has been obtained over the shorter duration of eight periods of the fundamental mode of secondary instability. The differences between these two curves in the downstream part of the streamwise region indicate the onset of stochastic solution behavior as the laminar flow begins to break down. In the upstream region $(x / c<0.51)$ when the wave amplitude is small $\left(A / U_{\infty}<0.5 \%\right)$ the instability undergoes linear growth with the growth rate comparing well with that based on the secondary instability analysis. Following a small region of linear growth, the growth rate of the secondary instability wave decreases as a result of nonlinear effects. Consequently, the evolution of the fundamental amplitude 
as derived from the DNS solution begins to deviate from the linear predictions near $x / c=0.515$. The growth rate has reduced appreciably near $x / c=0.53$ so that the evolution of fundamental amplitude over $0.53<x / c<0.54$ indicates a quasi-saturated behavior. The time history of streamwise velocity perturbation at the locations of modal peak within this region confirms that the initially sinusoidal time dependence of the unsteady perturbations has acquired a distorted signal shape with a significant higher harmonic content.

The quasi-saturation behavior is followed by an abrupt increase in the modal amplitude near $x / c=0.545$ and the signal develops a significant degree of randomness as indicated by the variance in model amplitudes from two different portions of time evolution. For reasons that are yet to be determined, the extent of the quasi-saturated region appears to be somewhat longer than that predicted by the previous nonlinear PSE computation. ${ }^{6}$

At $x / c \approx 0.554$, the modal amplitude reaches a maximum level of $A_{\max } / U_{\infty} \approx 3.5 \%$. Further downstream, the modal amplitude decreases, indicating the breakdown of the instability wave. Starting from $x / c \approx 0.565$, the wave amplitude becomes nearly uniform, indicating that the flow is likely to be turbulent at this stage.

Figure 4 displays the streamwise development of skin friction coefficient averaged in time and spanwise coordinate. The skin friction maintains a laminar level until $x / c \approx 0.554$, where it starts to rise rapidly, indicating the onset of transition. The onset of transition approximately coincides with the peak location of the modal amplitude of the secondary instability mode shown in Figure 3. At a location slightly downstream of the onset $(x / c \approx 0.564)$, the skin friction reaches a maximum value that is close to ten times the laminar level. For a transition triggered by a secondary high-frequency instability, the experiments at DLR ${ }^{26}$ found that the rise in $C_{f}$ undergoes an explosive growth rather than a gradual broadening of the Fourier spectrum of the unsteady disturbance motion, and so the abrupt rise in $C_{f}$ in the current simulation appears consistent with the experimental observations. Moreover, the overlap between the region with a rapid $C_{f}$ rise and the region with a reduction in the fundamental amplitude of the secondary instability wave (Figure 3) suggests that the latter might be a good indicator for locating transition onset. At $x / c \approx 0.569$, the mean chordwise velocity profile relaxes to a fully turbulent profile that shows a (narrow) logarithmic region and, furthermore, conforms well with the incompressible law-of-the-wall profile following the van Driest transformation (Figure 5).

To show the spanwise distribution of the transition front, contours of time-averaged wall shear distribution are plotted in Figure 6a. The mean wall-shear contours clearly display a noticeable nonuniformity in the spanwise direction. To better visualize the transition-front pattern, the contours of wall shear are periodically extended in the spanwise direction and black dashed lines are added to mark the onset of increased skin friction (Figure 6b). The transition front exhibits a sawtooth pattern. Similarly shaped transition fronts have been noted during surface flow visualization of swept wing boundary layers when the transition process is dominated by stationary crossflow instability (as opposed to traveling modes, which lead to smoother spanwise variations in the transition front). ${ }^{27}$ The transition-front pattern can be further visualized by near-wall turbulence structures. Figure 7 displays a top view of the contours of chordwise velocity in a streamwise-spanwise plane corresponding to $z^{+} \approx 15$ at $x / c=0.569$. Similar to the wall shear, the boundary dividing the non-turbulent region from turbulent region shows a similar sawtooth pattern. For $x / c<0.554$, the near-wall region is free of turbulent fluctuations; and the velocity maintains a laminar level with lower values. Turbulent fluctuations start at $x / c \approx 0.554$, turbulent structures start to appear at particular spanwise locations and spread to adjacent spanwise locations farther downstream. The whole spanwise domain becomes uniformly turbulent starting from $x / c \approx 0.564$.

To track the evolution of flow structures from the laminar region through the process of transition to turbulent flow, Figure 8 shows the mean and time-RMS of chordwise velocity at successively downstream locations. At a pre-transition station of $x / c=0.524$ (Fig. 8a), the contour shapes are relatively similar to those prescribed at the inlet (Figure 2), and the mode shapes of the stationary crossflow (white line contours) and the 'Y-Mode' secondary instability (color contours) can still be clearly identified. Somewhat further downstream $(x / c=0.530$ to $x / c=0.546)$, the fluctuation contours get progressively distorted near the peak of the secondary instability mode shape. At the transition onset, $x / c=0.554$, where the mean skin friction starts to rise, the overturning of the crossflow vortex becomes more diffused, affecting a larger cross-sectional area. Moreover, smaller chaotic structures start to appear within the boundary layer and propagate to the wall in some portions of the span, while in other portions of the span the flow remains laminar. Within the transitional region where $C_{f}$ undergoes rapid rise $(x / c=0.560)$, even more small structures appear and propagate towards the surface (Fig. 8b). Further downstream, a larger portion of 
span are populated by smaller scale structures. Characterized by localized strong events, as indicated by pockets of large fluctuations, the near-wall flow remains highly non-uniform in the spanwise direction. At $x / c=0.564$ where the skin friction obtains its maximum value, the mean crossflow vortex contours have lost the prominent overturning feature, the peak fluctuations have moved much closer to the wall, and the near-wall flow field is populated by small vortical structures across the span. As a result, the whole surface is exposed to downwash induced by the near-wall vortices. The vorticity generated due to the breakdown process remains concentrated near the wall, resulting in maximum turbulent drag. Although the small scales become nearly uniform across the span, inhomogeneity remains in large scale motions. Farther downstream into the fully turbulent region $(x / c=0.57)$, the near wall flow has become relatively homogeneous. However, memory of the initial stationary vortex persists as indicated by the spanwise inhomogeneity of the large-scale motions within the main part of the boundary layer.

To further visualize the flow structures during the breakdown stage, Fig. 9 plots the contours of instantaneous chordwise velocity in a streamwise wall-normal plane. In the laminar region, secondary instability is concentrated near the edge of the boundary layer. At $x / c \approx 0.554$, where $C_{f}$ starts to rise rapidly, the secondary instability begins to break down. Further downstream corresponding to the region where $C_{f}$ rises rapidly, the fluctuation moves progressively towards the surface. At $x / c \approx 0.564$ where $C_{f}$ has reached its maximum, the fluctuation has propagated to the near-wall region and the whole boundary layer shows evidence of turbulence. The propagation of fluctuations towards the surface can be further shown statistically by the $x-z$ contours of the time-averaged turbulent kinetic energy (TKE) at selected spanwise locations (Fig. 10). Similar to the flow visualization, high TKE regions are concentrated near the boundary layer edge at pre-transition locations. They propogate to the wall during the laminar breakdown and high TKE levels are achieved nearly throughout the whole boundary layer after transition.

Figure 11 provides a three-dimensional view of the instantaneous chordwise velocity to visualize the secondary instability and laminar breakdown of stationary crossflow vortices. Without the secondary instability wave, the isosurface would have been a tubular shape approximately aligned with the inviscid streamline, representing the primary crossflow vortex. As the secondary instability grows, small rib-like structures develop on top of the tubular structure. These structures are aligned at an oblique angle to the axis of the crossflow vortex. Farther downstream, even smaller structures emerge and the laminar breakdown process ensues.

\section{Concluding Remarks}

Direct numerical simulation of transition due to secondary instability of stationary crossflow vortices in a swept wing boundary layer has been performed under finite Mach number for a realistic airfoil configuration that is relevant to subsonic transports with an extended region of natural laminar flow. Both linear and nonlinear growth of the secondary instability is observed and the laminar breakdown process is predicted by the DNS. During the transition process, the skin friction coefficient rises rather rapidly within a few wavelengths of the secondary instability mode. Rib-like structures are observed riding on top of the crossflow vortex during the initial stage of the transition. The wall shear distribution shows a sawtooth pattern that is analogous to the previously documented surface flow visualizations of transition due to stationary crossflow instability. The memory of the stationary crossflow vortex persists well into the turbulence region and this could influence the accuracy of overall drag predicted using purely Reynolds averaged Navier-Stokes equations. The downstream region of the flow is studied and fully turbulent features are observed.

Follow-on work will involve a scrutiny of the DNS database to ascertain the details of disturbance modification in the vicinity of the transition onset location, along with an assessment of the effects of numerical resolution on the details of the transition zone. The streamwise length required to establish a spanwise homogeneous turbulent state will also be explored.

\section{Acknowledgments}

This work was performed as part of the Aeronautical Sciences Project of NASA's Fundamental Aeronautics Program (FAP). The authors would like to thank Prof. Pino Martín of the University of Maryland for providing the original code which has been modified for the current study. The simulations have been conducted using the Pleiades Supercomputer of the NASA Advanced Supercomputing (NAS) Division. The authors also thank Dr. Minwei Wu for his assistance during the initial phase of these computations. 


\section{References}

${ }^{1}$ Washburn, A., "Drag Reduction Status and Plans Laminar Flow and AFC," Oral Presentation at AIAA Aero Sciences Meeting, Orlando, FL, 2011.

${ }^{2}$ Saric, W. S., Carpenter, A. L., and Reed, H. L., "Laminar Flow Control Flight Tests for Swept Wings: Strategies for LFC," AIAA Paper 2008-3834, 2008.

${ }^{3}$ Carpenter, A. L., Saric, W. S., Reed, H. L., and Saric, W. S., "Laminar Flow Control on a Swept Wing with Distributed Roughness," AIAA Paper 2008-7335, 2008.

${ }^{4}$ Rhodes, R. G., Carpenter, A. L., Reed, H. L., and Saric, W. S., "CFD Analysis of Flight-Test Configuration for LFC on Swept Wings," AIAA Paper 2008-7336, 2008.

${ }^{5}$ Belisle, M. J., Neale, T. P., Reed, H. L., and Saric, W. S., "Design of a Swept-Wing Laminar Flow Control Flight Experiment for Transonic Aircraft," AIAA Paper 2010-4381, 2010.

${ }^{6}$ Li, F., Choudhari, M. M., Carpenter, M. H., Malik, M. R., Chang, C. L., and Streett, C. L., "Roughness Based Crossflow Transition Control for a Swept Airfoil Design Relevant to Subsonic Transports," AIAA Paper 2010-4380, 2010.

${ }^{7} \mathrm{Wu}$, X. and Moin, P., "Direct Numerical Simulation of Turbulence in a Nominally Zero-Pressure-Gradient Flat-Plate Boundary Layer," Journal of Fluid Mechanics, Vol. 630, 2009, pp. 5-41.

${ }^{8}$ Jiang, L., Choudhari, M. M., Chang, C. L., and Liu, C., "Numerical Simulations of Laminar-Turbulent Transition in Supersonic Boundary Layer," AIAA Paper 2006-3224, 2006.

${ }^{9}$ Mayer, C. S. J., Terzi, D. V., and Fasel, H. F., "Direct Numerical Simulation of Complete Transition to Turbulence via Oblique Breakdown at Mach 3," Journal of Fluid Mechanics, Vol. 674, 2011, pp. 5-42.

${ }^{10}$ Wassermann, P. and Kloker, M., "Mechanisms and Passive Control of Crossflow-Vortex-Induced Transition in a ThreeDimensional Boundary Layer," Journal of Fluid Mechanics, Vol. 456, 2002, pp. 49-84.

${ }^{11}$ Li, F., Choudhari, M. M., Chang, C. L., Streett, C. L., and Carpenter, M. R., "Roughness Based Crossflow Transition Control: A Computational Assessment," AIAA Paper 2009-4105, 2009.

${ }^{12}$ Malik, M. R., Li, F., Choudhari, M. M., and Chang, C. L., "Secondary Instability of Crossflow Vortices and Swept-Wing Boundary-Layer Transition," Journal of Fluid Mechanics, Vol. 399, pp. 85-115.

${ }^{13}$ Chang, C. L., "Langley Stability and Transition Analysis Code (LASTRAC) Version 1.2 User Manual," Tech. rep., NASA/TM-2004- 213233, 2004.

${ }^{14} \mathrm{Li}$, F. and Choudhari, M. M., "Spatially Developing Secondary Instabilities and Attachment Line Instability in Supersonic Boundary Layers," AIAA Paper 2008-590, 2008.

${ }^{15}$ Jiang, L., Choudhari, M. M., Chang, C. L., and Liu, C., "Direct Numerical Simulations of Crossflow Disturbances in Supersonic Boundary Layers," AIAA Paper 2004-589, 2004.

${ }^{16}$ Martín, M., "DNS of Hypersonic Turbulent Boundary Layers. Part I: Initialization and Comparison with Experiments," Journal of Fluid Mechanics, Vol. 570, 2007, pp. 347-364.

${ }^{17}$ Duan, L., Beekman, I., and Martín, M. P., "Direct Numerical Simulation of Hypersonic Turbulent Boundary Layers. Part 3: Effect of Mach Number," Journal of Fluid Mechanics, Vol. 672, 2011, pp. 245-267.

${ }^{18}$ Duan, L., Beekman, I., and Martín, M. P., "Direct Numerical Simulation of Hypersonic Turbulent Boundary Layers. Part 2: Effect of Wall Temperature," Journal of Fluid Mechanics, Vol. 655, 2010, pp. 419-445.

${ }^{19}$ Duan, L. and Martín, M. P., "Direct Numerical Simulation of Hypersonic Turbulent Boundary Layers. Part 4: Effect of High Enthalpy," Journal of Fluid Mechanics, Vol. 684, 2011, pp. 25-59.

${ }^{20}$ Martín, M. P., Taylor, E. M., Wu, M., and Weirs, V. G., "A Bandwidth-Optimized WENO Scheme for the Direct Numerical Simulation of Compressible Turbulence," Journal of Computational Physics, Vol. 220, No. 1, 2006, pp. 270-289.

${ }^{21}$ Taylor, E. M., Wu, M., and Martín, M. P., "Optimization of Nonlinear Error Sources for Weighted Non-Oscillatory Methods in Direct Numerical Simulations of Compressible Turbulence," Journal of Computational Physics, Vol. 223, No. 1, 2006, pp. 384-397.

${ }^{22}$ Williamson, J., "Low-Storage Runge-Kutta Schemes," Journal of Computational Physics, Vol. 35, No. 1, 1980 , pp. 48-56.

${ }^{23}$ Pruett, C. D., Zang, T. A., Chang, C. L., and Carpenter, M. H., "Spatial Direct Numerical Simulation of High-Speed Boundary-Layer Flows part I: Algorithmic Considerations and Validation," Theoretical and Computational Fluid Dynamics, Vol. 7, No. 1, 1995, pp. 49-76.

${ }^{24}$ Pruett, C. D., Chang, C. L., and Streett, C. L., "Simulation of Crossflow Instability on a Supersonic Highly Swept Wing," Computers and Fluids, Vol. 29, 2000, pp. 33-62.

${ }^{25}$ Thompson, K. W., "Time Dependent Boundary Conditions for Hyperbolic Systems," Journal of Computational Physics, Vol. 68 , No. 1.

${ }^{26}$ Bippes, H., "Basic Experiments on Transition in Three-Dimensional Boundary Layers Dominated by Crossflow Instability," Progress in Aerospace Sciences, Vol. 35, No. 4, 1999, pp. 363-412.

${ }^{27}$ Reibert, M. S., Nonlinear Stability, Saturation, and Transition in Crossflow-Dominated Boundary Layers, Ph.D. thesis, Arizona State University, 1996. 


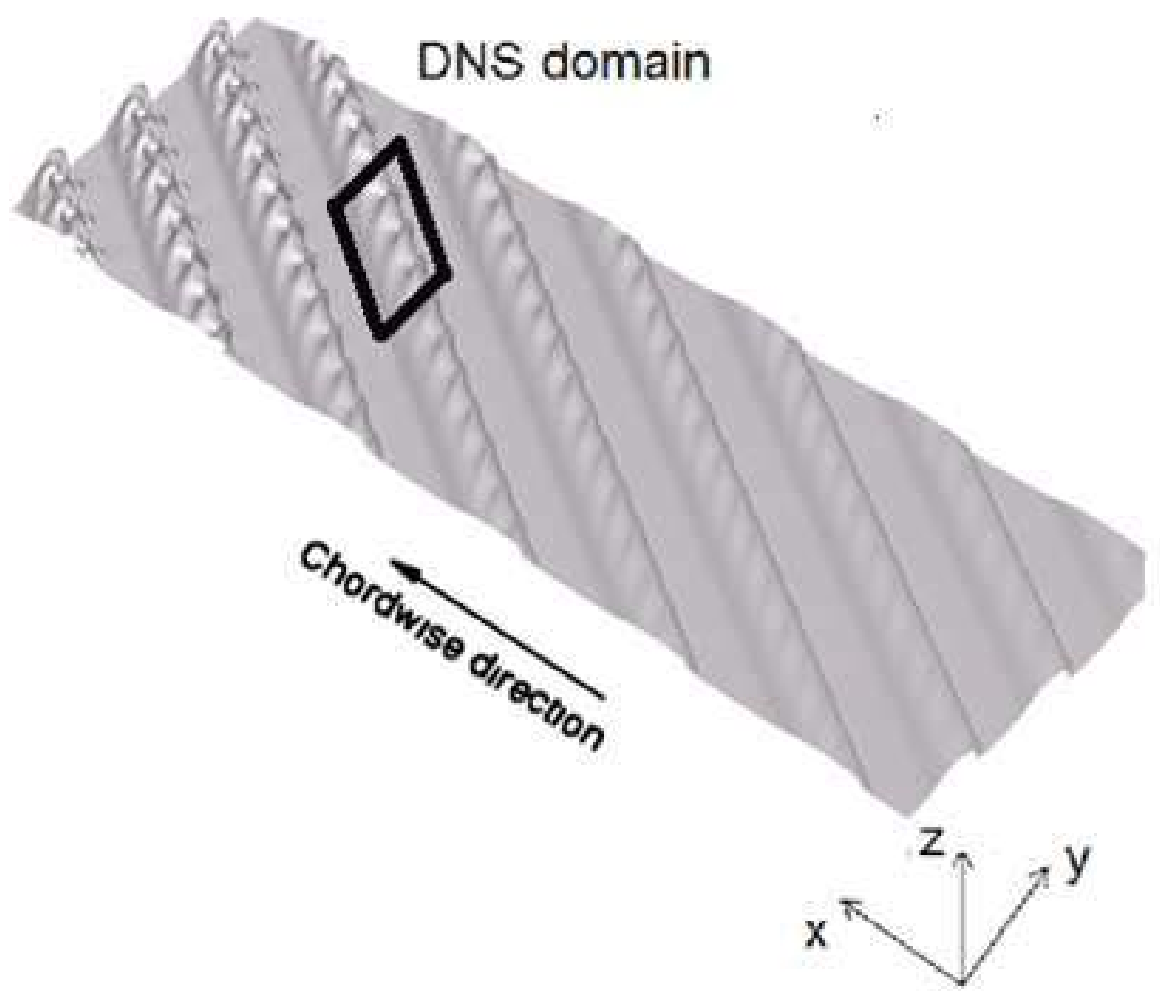

Figure 1. Sketch of the computational domain for the DNS simulation. The black frame labeled as DNS domain indicates the boundaries of the domain along the surface of the airfoil such that both the upstream and downstream boundaries of the domain are parallel to the airfoil leading edge (i.e., $y$ axis of the Cartesian frame); the underlying cylindrical structures represent the stationary pattern of crossflow vortex instability and the smaller, ribbed structures superposed on this pattern indicate an instantaneous view of the secondary instability waves developing over the stationary vortices. 


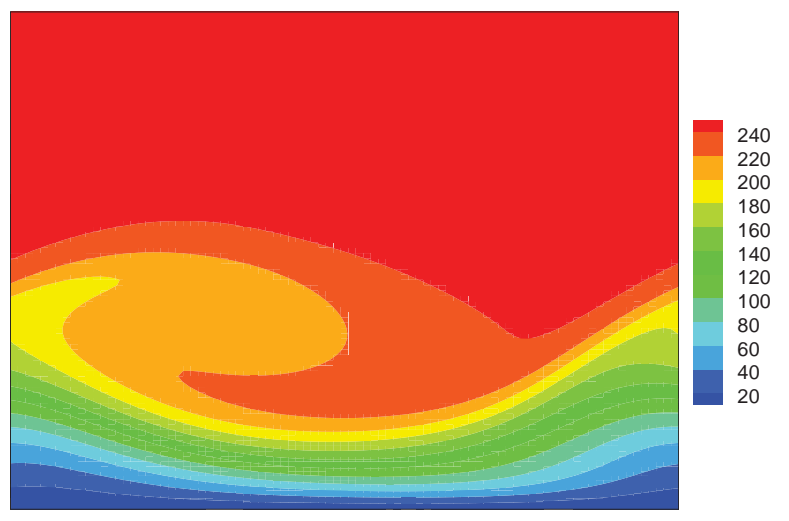

(a) Stationary crossflow vortex

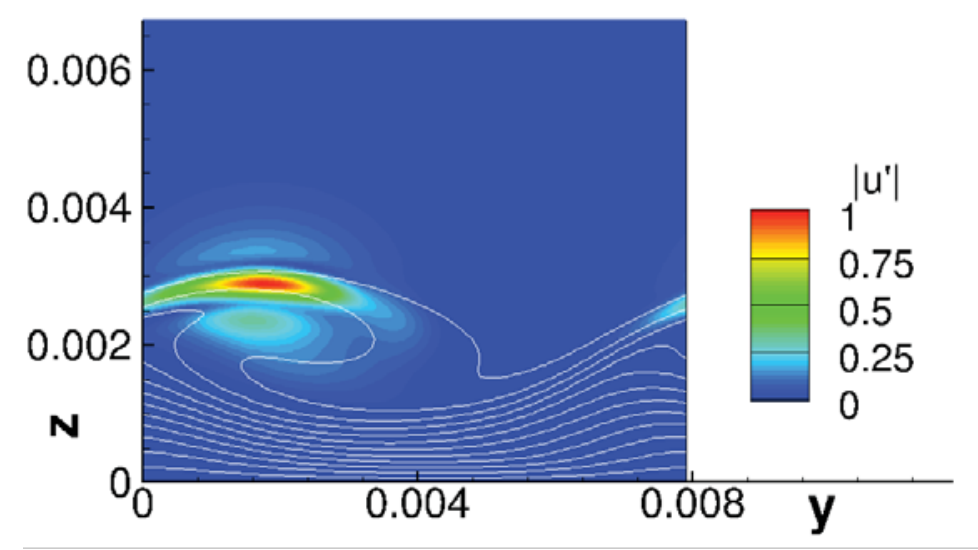

(b) 'Y-Mode' secondary instability

Figure 2. (a) Mean and (b) time-RMS distributions of chordwise velocity $\left(u_{x}\right)$ at the inlet of the DNS simulation. The white lines in the background of part (b) are the contours of mean chordwise velocity. 


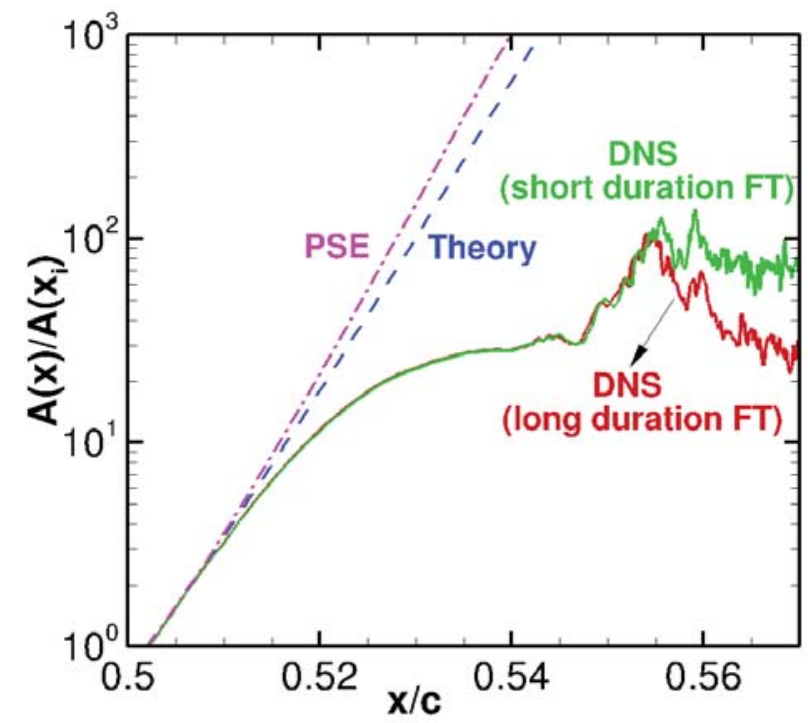

Figure 3. Chordwise development of the maximum chordwise velocity disturbance of fundamental mode. The green and red curves correspond to DNS results representing Fourier transforms computed over a short duration (eight periods of fundamental secondary mode) and long duration (more than 100 periods of the fundamental secondary mode), respectively; the blue dashed curve denotes prediction of the secondary instability theory; ${ }^{6}$ and the purple dash-dot curve denotes the linear PSE computation. ${ }^{6}$

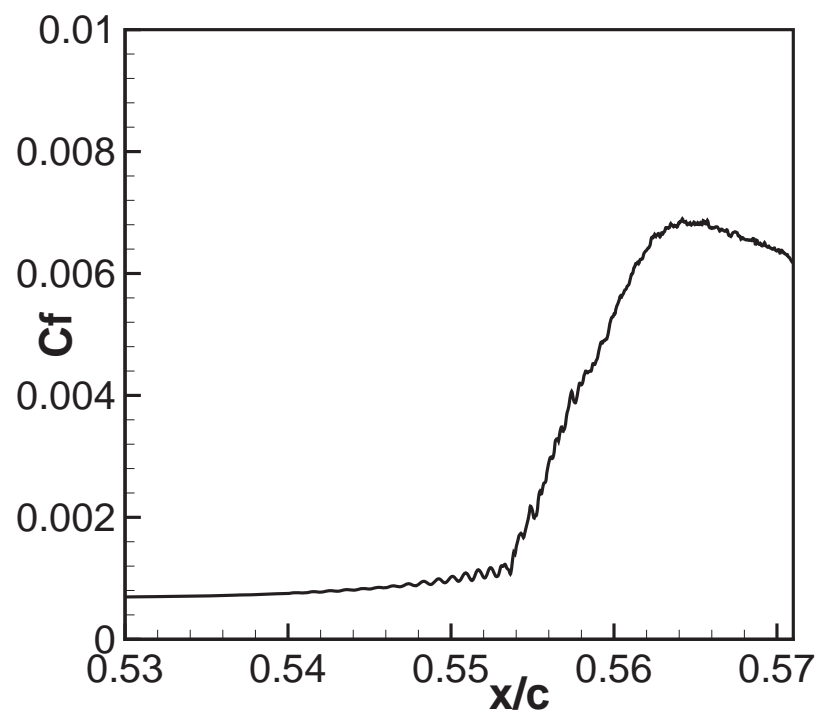

Figure 4. Evolution of skin friction coefficient averaged in time and span along the chordwise direction. 


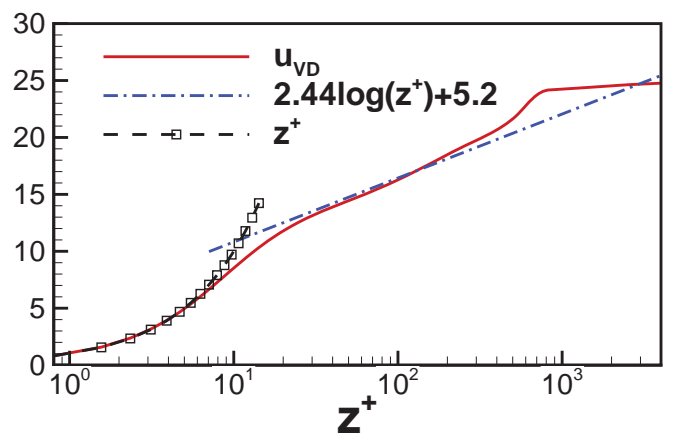

Figure 5. Van-Driest transformed chordwise velocity, $\bar{u}_{V D}$, at $x / c=0.568$, with $\bar{u}_{V D} \equiv$ $\left(1 / u_{\tau}\right) \int_{0}^{u_{x}}\left(\bar{T}_{w} / \bar{T}\right)^{1 / 2} d u_{x}$.

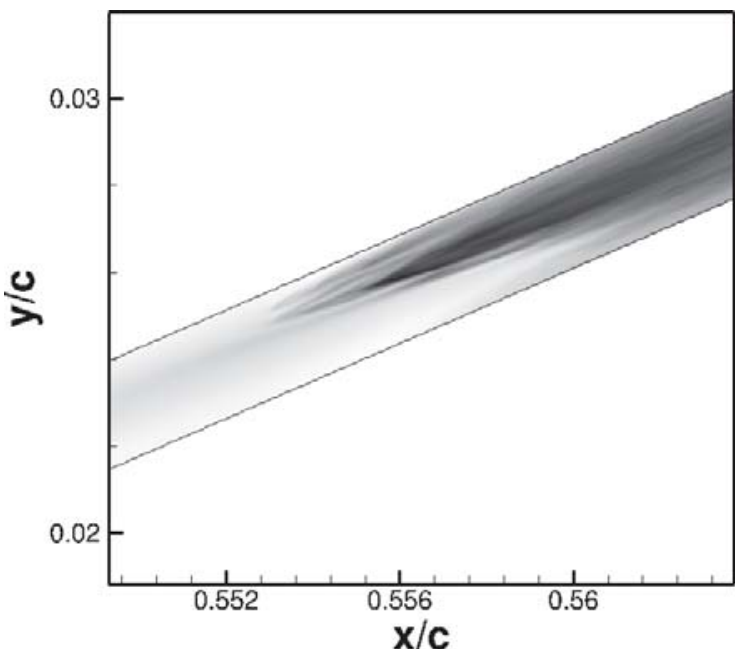

(a)

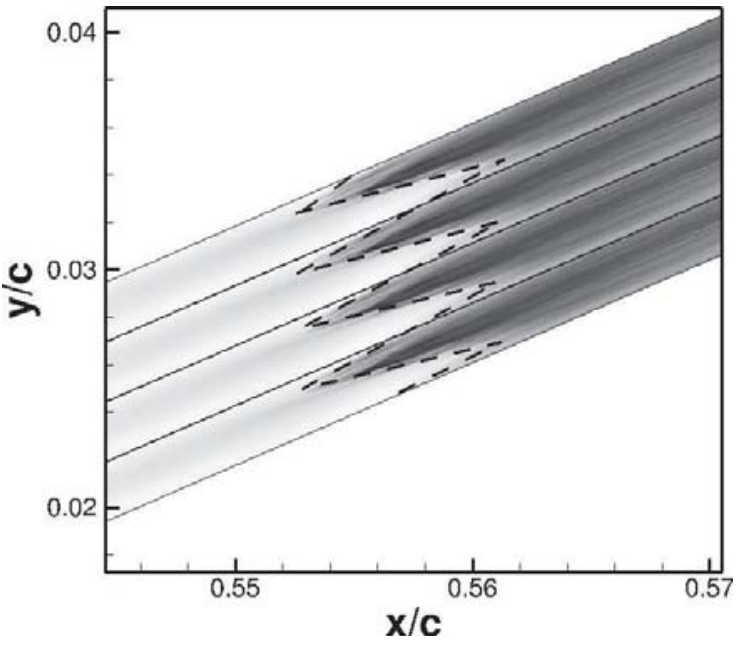

(b)

Figure 6. (a) Contours of time-averaged wall shear from the DNS, viewed in the x-y plane, with black color indicating high shear and white color indicating low shear; $(b)$ Multiple copies of ( $a$ ) duplicated periodically in the spanwise direction. Black dash lines indicate the saw tooth curve marking the onset of increased skin friction. 


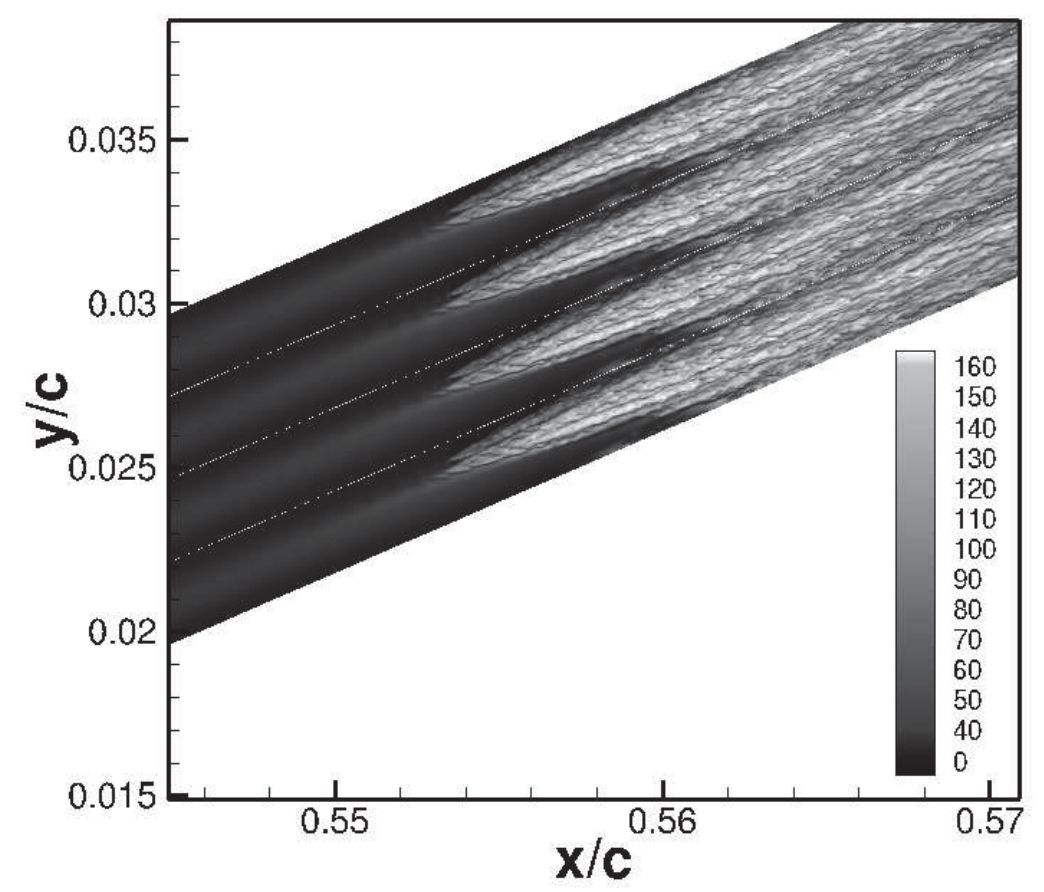

Figure 7. Contours of chordwise velocity $\left(u_{x}\right)$ at $z^{+} \approx 15$, with the viscous length $z_{\tau}$ chosen to be that at $x / c \approx 0.569$. 


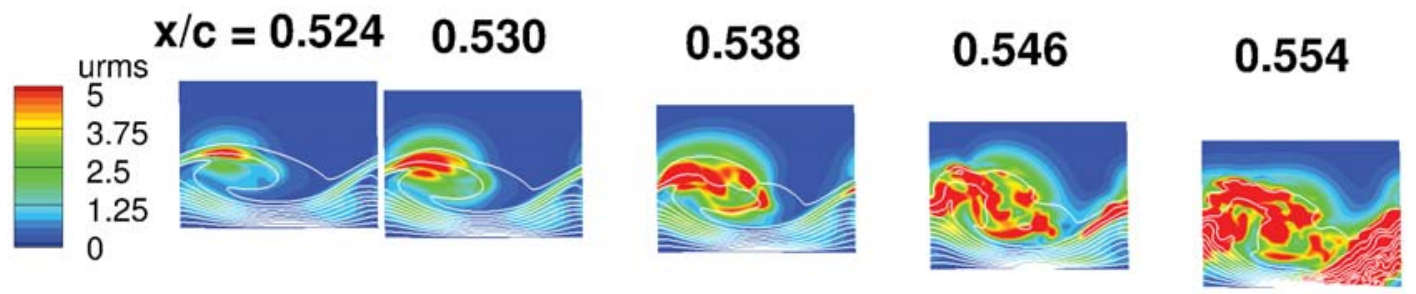

(a) $x / c \leq 0.554$ (i.e., upstream of chordwise location corresponding to rapid rise in average skin friction)

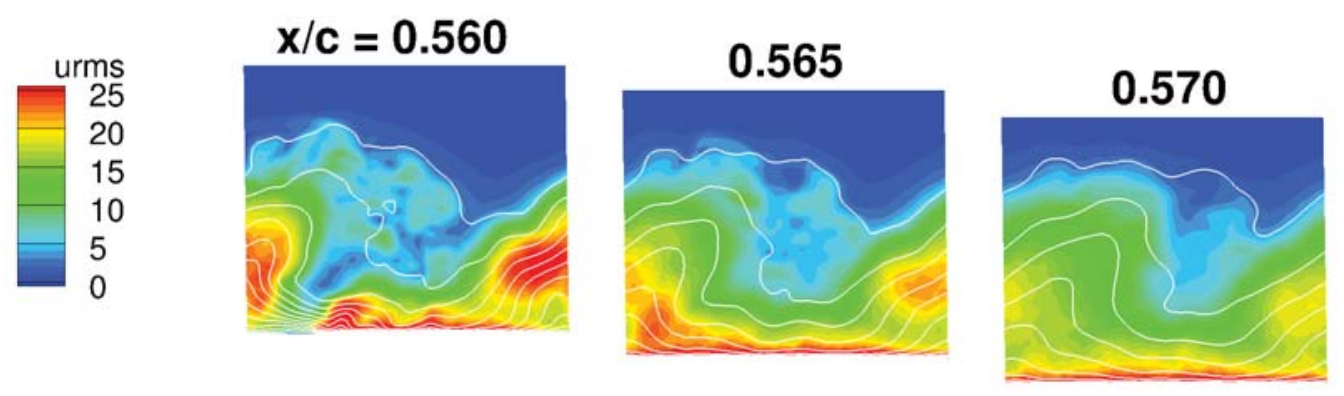

(b) $x / c>0.554$

Figure 8. Time-RMS distributions of chordwise velocity at selected chordwise stations. The white lines in the background are the contours of mean chordwise velocity. $x / c$ increasing from left to right.

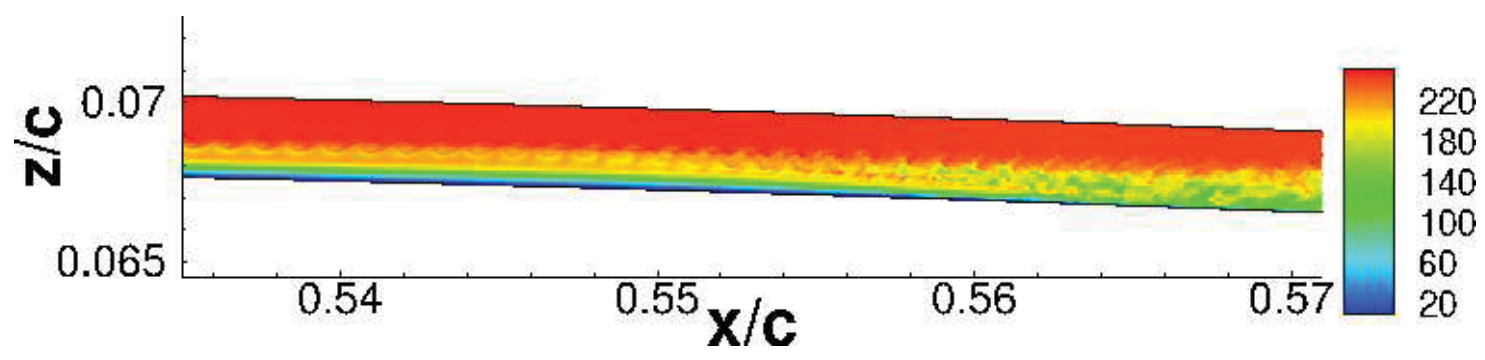

Figure 9. Instantaneous chordwise velocity $\left(u_{x}\right)$ contours in a chordwise wall-normal plane from the DNS. The flow direction goes from left to right. 


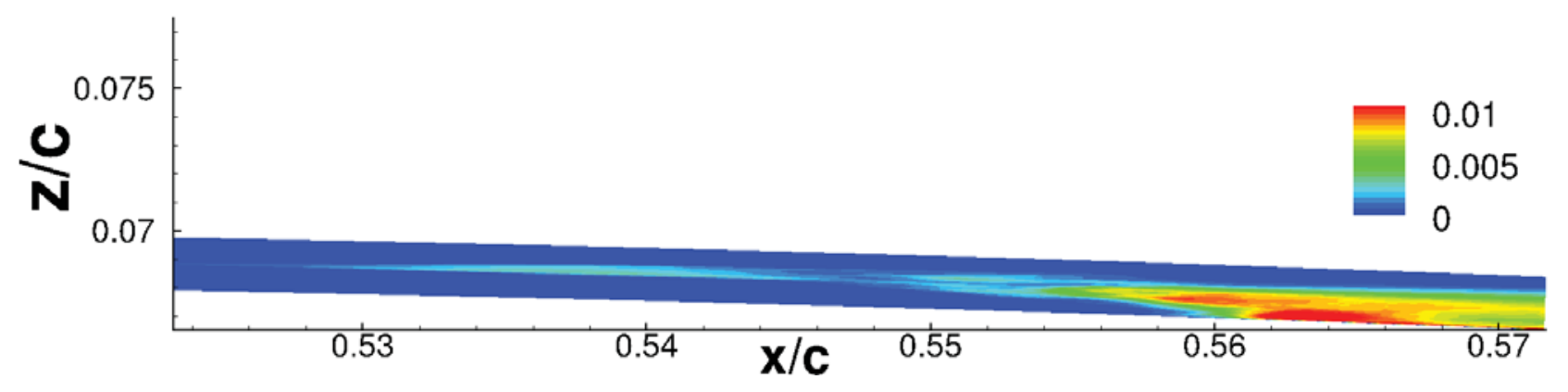

Figure 10. Time-RMS distributions of turbulent kinetic energy ( $T K E$ ) in a chordwise wall-normal plane from the DNS. The flow direction goes from left to right. 


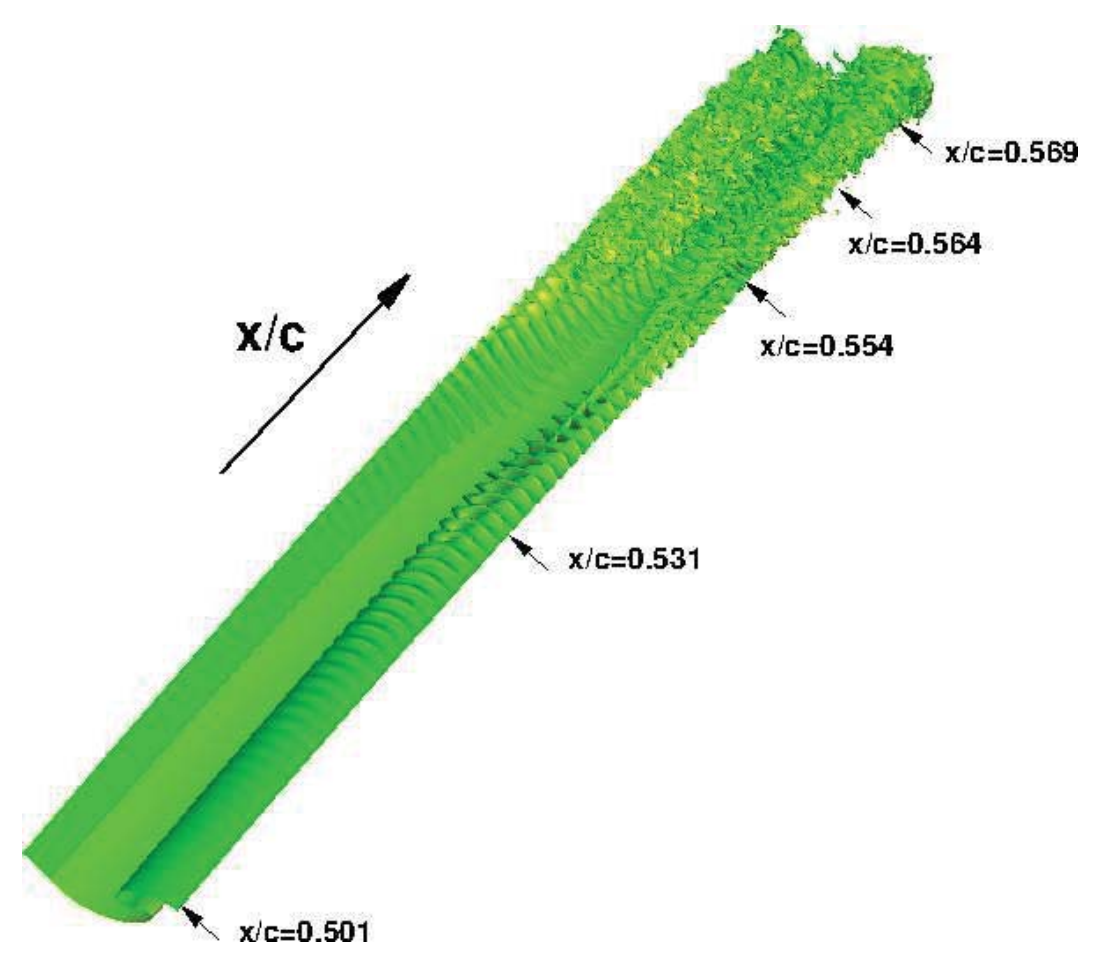

(a) Overall view

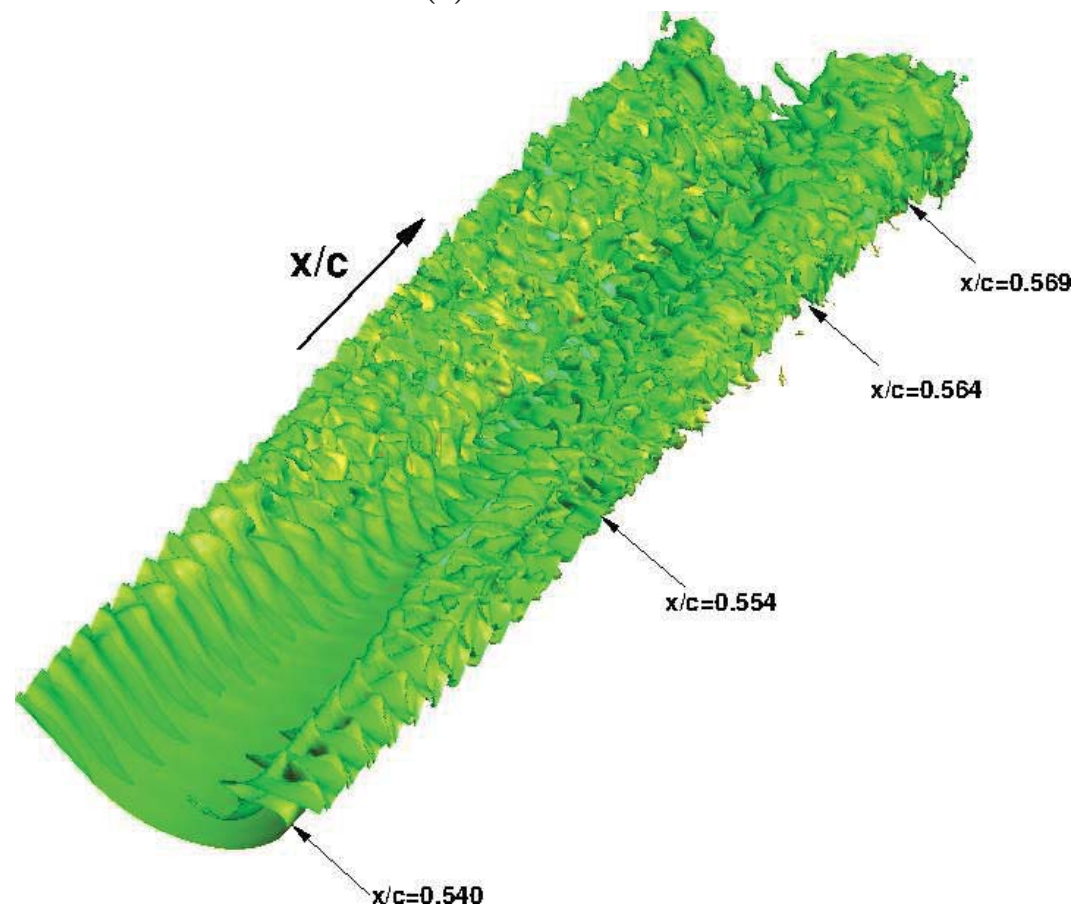

(b) Zoomed in view

Figure 11. Iso-surface of chordwise velocity $\left(u_{x}\right)$ corresponding to 85 percent of freestream speed. 